\title{
Index for Volume 17 of Molecular Plant-Microbe Interactions
}

\section{AUTHOR AND SUBJECT INDEX}

Achouak, W., 872

Acidovorax avenae, on rice, cells elicit immune response, 986

Agrobacterium tumefaciens, butyrolactone assimilation, lactone signal, 951

Ahren, D., 202

Aivalakis, G., 1283

Alabouvette, C., 1201

Alarcón-Chaidez, F., 162

Albersheim, P., 888

Albugo candida, on Arabidopsis thaliana

-interaction phenotypes, cover photo, July

-resistance gene $R A C 1$, other gene dependence, 711

Alfalfa, symbiotic abnormalities, MsLEC1 transgenes, 16

Alfano, J. R., 184, 447

Allen, C., 686, 696

Almeida, N. F., Jr., 827

Alonso, J. M., 696

Alvarez, M. E., 343

Ampe, F., 292

Amrein, H., 90

Anand, A., 1306

Andrie, R. M., 491

Angell, S., 1086

Antibiotic, 2,4-diacetylphloroglucinol, Pseudomonas fluorescens, Fusarium oxysporum response, 1201

Apoptosis

-Nicotiana benthamiana and tomato, Pseudomonas type III effector, 1328

-oat defense response, hypersensitivity and nitric oxide and oxygen, 245

-wheat line extracts, pathogenesis-related proteins, 1306

Arabidopsis sp.: broad spectrum resistance, rhizobacteria-induced, transcriptome, 895;

Colletotrichum pathosystem, molecular dissection of interaction, 272; GSTF8 promoter, Rhizoctonia solani, 70; mitochondria, harpin inactivation, 131; salicylic acid-responsive genes, NPR1independent activation, 34

-A. thaliana: Albugo candida, resistance conferred by gene $R A C 1$, other genes, 711 ; Colletotrichum higginsianum on, resistance from RCJI locus, 749; Fusarium oxysporum, resistance, ethylene response factor, 763; gene up-regulated by pathogen, enhancer trapping, 1086; hypersensitivity, feedback control, 357; incompatible interactions, proline accumulation and AtP5CS2 gene activation, 343; Pseudomonas

brassicacearum, phenotypic variation, colonization strategy, 872; Ralstonia solanacearum, flagellin as elicitor, 696; Spring beauty latent virus, SSB1 locus, 967; victorin sensitivity, characterization, 577

Arakane, Y., 1306

Aranda, M. A., 668

Ardales, E. Y., 771

Atkinson, I., 1051

Avrova, A. O., 943

Ayliffe, M. A., 853

Ayme, V., 322

Azukawa, Y., 967
Bacteria, plant-growth-promoting, canola root changes induced by, ACC-deaminase, 865

Bai, J., 771

Balaji, V., 1212

Balázs, E., 837

Banba, M., 1223

Bao, Y., 583

Barley

- Bipolaris sorokiniana, hemibiotroph, symptomless interaction phenotype, 366

— mutant albostrians, powdery mildew resistance, 374

- powdery mildew: apoplastic $\mathrm{pH}$ signaling, 118; genes and papilla resistance, 729; inappropriate form of wheat formae speciales, 304

-quantitative resistance, germinlike protein GLP4, 109

-Rhynchosporium secalis, virulence mechanisms, genetic analysis, 1114

- spot blotch and powdery mildew, cell wall penetration, cover photo, April

-wheat powdery mildew on: inappropriate host, cover photo, March; inappropriate pathogen, apoptosis suppressor homologue, 484

Batut, J., 235, 292

Bäumer, N., 383

Bäumlein, H., 62

Bean (see also Phaseolus vulgaris)

-Pseudomonas putida, systemic resistance induced, lipoxygenase pathway, 1009

Becana, M., 1294

Becker, A., 292

Beer, S. V., 644

Bell, K. S., 943

Bellogín, R. A., 676

Bemisia tabaci, on tomato, $M i$-mediated resistance, 532

Bender, C. L., 162

Bent, A. F., 511, 696

Bergès, H., 292

Bergman, B., 436

Bergmann, C. W., 888

Berrocal-Lobo, M., 763

Bes, M., 414

Betsuyaku, S., 245

Betula pendula, ectomycorrhizal root tissue, transcriptional response, 202

Beynon, J. L., 711

Bhatterai, E., 1337

Bioimaging, phytoplasma detection, plant distribution, 1175

Biological control, Pseudomonas chlororaphis, phenazine-1-carboxamide production, factors, 557

Bioremediation, rhizoremediation, plantmicrobe interaction, review, 6

Bipolaris sorokiniana, on barley

-cell wall penetration, cover photo, April

- symptomless interaction phenotype, 366

Birch, P. R. J., 943

Blanco, F., 34

Bloemberg, G. V., 6, 557

Blumeria graminis

-on barley: apoplastic $\mathrm{pH}$ response, cover photo, January; apoplastic pH signaling, 118;
ERRATA PAGE NUMBERS IN ITALICS

cell wall penetration, cover photo, April; genes and papilla resistance, 729;

inappropriate pathogen, apoptosis suppressor homologue, 484; mutant strain, enhanced resistance, 374

-on barley and wheat: nonhost interaction with inapppropriate formae speciales, 304;

quantitative resistance and germinlike protein GLP4, 109

Bockus, W. W., 1306

Bolaños, L., 216

Bonas, U., 805, 1039

Bonilla, I., 216

Bonshtien, A., 1212

Boot, K. J. M., 816

Borgonie, G., 846

Borhan, M. H., 711

Bormann, C. A., 1126

Börner, T., 374

Botrytis cinerea, Ga subunit, genes upregulated during infection, 537

Boucher, C., 931

Boyd, L. A., 1242

Branch, C., 351

Bressan, R. A., 780

Brewin, N. J., 216

Brill, L. M., 16

Brooks, D. M., 162

Broughon, W. J., 958, 1153

Brown, D., 686

Brown, I., 805, 1039

Bruand, C., 292

Brumbley, S. M., 175, 827

Bruno, K., 547

Bucciarelli, B., 1294

Buhr, K., 1126

Burdman, S., 593, 602

Burns, J. K., 1394

Burse, A., 43

Camacho, M., 676

Camargo, L. E. A., 827

Camarotte, G., 827

Candy, J. M., 175

Canola, gene expression changes, induced by plant-growth-promoting bacteria, 865

Cao, M., 394

Cao, Y., 633

Capela, D., 292

Capsicum annuum (see also Pepper)

-recessive resistances, genome-linked protein, 322

Caranta, C., 322

Carkaci-Salli, N., 577

Carlier, A., 951

Carlson, R. W., 283

Carraro, D. M., 827

Carrer, H., 827

Carter, S. A., 583

Carver, T. L. W., 729

Castillo Ruiz, R. A., 1126

Cauliflower mosaic virus

- avirulence domain, viral virulence determination, 475

Cell death, lesion-mimic, rice and (At)NPR1, 140

Cermola, M., 720 
Chang, H.-S., 895

Chapman, B. P., 1051

Chatagnier, O., 1385

Chatterjee, A. K., 184

Che, F.-S., 986

Chemotaxis, Pseudomonas fluorescens to

Fusarium oxysporum, fusaric acid, 1185

Chen, C., 404

Chen, J., 447

Chen, W., 70

Cheng, N.-H., 583

Chern, M.-S., 140

Chevalier, S., 1269

Chevrot, R., 951

Chin-A-Woeng, T. F. C., 557

Chiurazzi, M., 1043

Christensen, A. B., 109

Christensen, N. M., 1175

Chung, K-R., 1394

Cillo, F., 98

Citrus, anthracnose, phytohormones and genes, 1394

Ciuffetti, L. M., 467, 491

Claeys, M., 846

Clavibacter michiganense, on tomato, proteomic analysis, resistance loci, 1019

Claviceps purpurea, on rye, oxidative stress response, transcription factor, 383

Clay, R. P., 888

Clemente, M. R., 1294

Cluzet, S., 909

Coaker, G. L., 1019

Cohen, V., 872

Cole, A. B., 976

Colletotrichum sp., on Arabidopsis, pathosystem, molecular dissection of interaction, 272

-C. acutatum, on citrus, phytohormones and genes, 1394

-C. higginsianum, on Arabidopsis, resistance from $R C J 1$ locus, 749

- C. lagenarium, cAMP-PKA signaling and Cmk1 MAP kinase, 1355

-C. trifolii, on Medicago truncatula, compatible and incompatible interaction, analysis, 909

Collmer, A., 184, 447, 644

Combier, J.-P., 1029

Conrod, S., 872

Contributors, information, 124

Cooksey, D. A., 999

Coomans, A., 846

Cooper, R. M., 456

Corn (see Maize)

Costet, L., 414

Cotton

-Fusarium infection, profile change response to infection, gene expression, 654

-polyploid, NBS-LRR-encoding resistance gene, 1234

Cousin, A., 414

Coutinho, L. L., 827

Coutts, K., 1086

Couvreu, M., 846

Covaleda, L., 1234

Cover photo

-Albugo candida on Arabidopsis thaliana, interaction phenotypes, July

-barley: spot blotch and powdery mildew, fungal penetration, April; wheat powdery mildew, inappropriate fungus,

hypersensitivity, March

-Blumeria graminis, on barley, apoplastic $\mathrm{pH}$ response, January

- Hebeloma cylindrosporum, on Pinus pinaster roots, mutants, September

-Leifsonia xyli, circular genome, August

- phytoplasma, distribution in infected plants, bioimaging, November

-Pyrenophora tritici-repentis, host-selective toxin gene, schematic analysis, May

-Rhizobium etli, abortive nodule primordia, ectopic roots, October

-Tobacco mosaic virus, gene silencing delay, encoded suppressor, June

-Xanthomonas campestris, on tomato leaflets, symptoms, February

-Xanthomonas oryzae pv. oryzae, on rice, Xa5 resistance gene, December

Cucumber mosaic virus

—on Arabidopsis thaliana, resistance mediated by salicylic acid independence, 623

-necrotic pathotype, amino acid determinant, 837

-in tomato, RNA-mediated transgenic resistance, mechanisms, 98

Cucumis melo, Melon necrotic spot virus, resistance overcome, molecular characterization, 668

Cui, Y., 184

Damais, A., 414

Dardick, C., 593

Darvill, A., 888

da Silva, A. C. R., 827

da Silva, F. G., 593

Daykin, M., 880

Deakin, W. J., 958

Dean, R., 1337

Debbie, P., 1212

de Bruijn, F. J., 292

Delis, C., 1283

Delp, G., 1103

del Rosario Espuny, M., 676

De Meutter, J., 846

Deng, Y., 1139

Denny, T. P., 1376

de Oliveira, J. C. F., 827

de Oliveira, M. C., 827

Desbrosses, G., 789, 1283

Descombin, J., 1385

de Souza, A. A., 827

Dessaux, Y., 951, 1269

de Vos, C. H., 1201

de Weert, S., 1185

Diallo, E., 958

Díaz, J. A., 668

Dickman, M. B., 394

Dimou, M., 1283

Ding, X. S., 583

Divéki, Z., 837

do Amaral, A. M., 827

Dommes, J., 1009

Dong, Y., 1078, 1139

Doss, P., 739

D’Ovidio, R., 1402

Dowd, C., 654

Driscoll, B. T., 1318

Driskel, B. A., 739

Dröge-Laser, W., 1162

Du, C., 1234

Duby, F., 1009

Dudler, R., 90, 109

Dumas, B., 272, 909

Dunning, F. M., 696

Durner, J., 131

Dymov, S. I., 1318

Eardley, L., 633

Ebbole, D. J., 1337

Ecker, J. R., 696

Edel-Hermann, V., 1201

Efrose, R. C., 1283

Eichmann, R., 484

Ekman, M., 436

El-Dorry, H. A., 827
Elicitor

-flagellin, Ralstonia solanacearum on Arabidopsis thaliana, 696

-flagellin perception, cultured rice cells, 986

-harpin: Arabidopsis sp. mitochondrial cells,

131; Erwinia chrysanthemi, mutant analysis, 184

-Phytophthora sojae on soybean, avirulence and resistance gene, 394

—victorin: in Arabidopsis, 577; biosynthesis in oat, 81

Ellis, J., 224

Enju, A., 749

Enzymes

-ACC-deaminase, plant-growth-promoting bacteria, effect on canola roots, 865

- polygalacturonase, inhibitors and activators, Aspergillus niger and Phaseolus vulgaris, 888

Erlandsson, R., 202

Erratum, vol. 17, no. 7, 2004, 1039

Erwinia spp.

-E. amylovora, in Rosaceae, multidrug efflux pump, phytoalexin-inducible, 43

-E. carotovora: necrosis-inducing virulence protein, characterization, 1366; on potato, lactones and infection, 1269; on potato, pooled transposon mutation grid, effector and helper proteins, 943 ; on potato, susceptibility increased by lactones, 880

$-E$. chrysanthemi: gene cluster, Hrp type III system encoded, virulence genes, 644; mutants, strain-specific regulation, Hrp type III, 184; plant-upregulated genes, genomewide identification, 999

Esquerré-Tugayé, M.-T., 272, 909

Fabro, G., 343

Fauconnier, M.-L., 1009

Faure, D., 951, 1269

Favaron, F., 1402

Fedorova, M., 1294

Felle, H. H., 118

Fengler, K. A., 511

Ferraioli, S., 720, 1043

Ferro, E. S., 827

Ferro, M. I. T., 827

Filosa, S., 720

Finetti-Sialer, M. M., 98

Finnegan, J., 224

Firnhaber, C., 1063

Fischer, U., 1162

Fitzgerald, M. A., 140

Flax, rust, resistance gene $L$, tobacco transgenic, defense response, 224

Flemetakis, E., 1283

Foley, R. C., 70

Folimonov, A., 583

Fortnum, B. A., 1376

Fourment, J., 292

Fournier, J., 909

Francez-Charlot, A., 292

Francis, D. M., 1019

Fray, R. G., 880

Fridborg, I., 1086

Frost, D., 224

Frühling, M., 62

Frutos, R., 414

Fujisaki, K., 967

Fujishige, N. A., 16

Fujiwara, S., 986

Furlan, L. R., 827

Fusarium oxysporum

—on Arabidopsis, ethylene response factor, 763

-on cotton, profile change response to

infection, 654

—defense response to antibiotic, 1201

Gabriel, D. W., 414

Gagliardi, P. R., 827 
Gallitelli, D., 98

Gamas, P., 909

Gao, L.-L., 1103

Gautheron, N., 1201

Gay, G., 1029

Gebhardt, C., 1126

Gene

-Arabidopsis DND2, "defense, no death" phenotype, 511

-AtP5CS2, Arabidopsis and Pseudomonas interaction, proline accumulation, 343

-avrA, Ralstonia solanacearum and tobacco, 1093

-AvrBs3/PthA, virulence determinants, rice bacterial blight, 1192

- avrRpt2, Pseudomonas syringae, virulence and avirulence activities, 313

-avrRxo1, Xanthomonas oryzae, maize gene Rxol comparison, 771

-EC 16 hrp/hrc cluster, Erwinia chrysanthemi, 644

- expansin, Melilotus alba to Sinorhizobium meliloti, plant interaction, 613

- Mi-1, tomato, resistance to nematodes, aphids, and whitefly, 55

-NBS-LRR, analogue family, cotton, 1234

- papilla resistance, powdery mildew on barley, 729

-plant-upregulated, genome-wide, Erwinia chrysanthemi, 999

-RAC1, Arabidopsis, white rust resistance, 711

-Rps1b, Phytophthora sojae on soybean, elicitor and regulators, 394

-ToxB, Pyrenophora tritici-repentis, hostselective gene, 467

-TRI, Arabidopsis, up-regulation by pathogen, 1086

-typA, Sinorhizobium meliloti on Medicago truncatula, adaptation, 235

-VfLb29, Vicia faba, activated in mycorrhizal roots, 62

- xa5, rice resistance, 1348

—virus-induced, Panicum mosaic virus capside protein, nonhost plant, 263

Germundsson, A., 921

Gheysen, Godelieve, 846

Gheysen, Greetje, 846

Gianinazzi, S., 1385

Gianinazzi-Pearson, V., 1385

Gibly, A., 1212

Giglioti, E., 827

Gijzen, M., 1051

Gil-Serrano, A. M., 676

Giordano, W., 613

Gjetting, T., 109, 729

Glazebrook, J., 895

Glick, B. R., 865

Glomeromycota, on Medicago truncatula, endosymbiosis, transcriptome profiling, 1063

Glycin, antimicrobial protein, modulation by cation, 780

Goggin, F. L., 532

Goldman, G. H., 827

Goldman, M. H. S., 827

Gorovoits, R., 394

Granado, J., 90

Gray, S. M., 571

Guilbaud, L., 322

Gunnera, Nostoc symbiosis, cyanobacterial

genes, heterocysts and dinitrogen fixation, 436

Guo, M., 447

Gutsche, A. T., 357

Hackworth, C. A., 16

Hagihara, F., 967

Hakoyama, T., 1223

Ham, J. H., 184, 511, 644

Hansen, M., 1175
Hanstein, S., 118

Harakava, R., 827

Hardham, A., 224

Harel, A., 394

Hase, S., 623

Hasegawa, P. M., 780

Hata, S., 1223

Haynes, J. G., 283

He, L., 1234

Hebeloma cylindrosporum

-nonmycorrhizal mutants, insertional mutagenesis, 1029

-on Pinus pinaster roots, nonmycorrhizal mutants, cover photo, September

Heim, U., 62

Herbert, C., 272

Hermann, S. R., 175

Hernández-Guzmán, G., 162

Herrmann, A., 118

Hess, W., 374

Heterodera schachtii, dorsal pharyngeal gland, ubiquitin extension proteins, 846

Heulin, T., 872

Hidalgo, Å., 676

Hirayama, T., 749

Hirsch, A. M., 16, 613

Hohn, T., 475

Hohnjec, N., 1063

Holeva, M. C., 943

Holub, E. B., 711

Holuigue, L., 34

Hontzeas, N., 865

Hotson, A., 633

Hou, Y.-M., 583

Howie, J. A., 1242

Howles, P., 224

Huang, E., 428

Hückelhoven, R., 118, 304, 366, 374, 484

Huguet, T., 235, 909

Hulbert, S. H., 771, 853

Hutcheson, S. W., 254

Hutten, R. C. B., 428

Hwang, C.-F., 55, 351

Hyman, L. J., 880, 943

Hypersensitivity

- Arabidopsis thaliana, Pseudomonas syringae, feedback control, 357

-barley powdery mildew, from wheat, 484

-Colletotrichum higginsianum on Arabidopsis, resistance locus, 749

-oat defense response, nitric oxide and oxygen role, 245

- Pseudomonas syringae on Arabidopsis, mitochondria and harpin, 131

Iaccarino, M., 720

Ibeas, J. I., 780

Ibekwe, A. M., 999

Ikegami, M., 623

Imai, S., 81

Iniguez, A. L., 1078

Ishida, J., 749

Ishihara, A., 81

Ishihara, T., 623

Isogai, A., 986

Itou, A., 1223

Ivanov-Vangelov, K., 676

Iyer, A. S., 1348

Jackson, L. F., 1114

Jacobsen, E., 428

Jacquemond, M., 322

Jacquet, C., 909

Jain, S. K., 374

James, E. K., 1294

Jia, X., 447

Jin, Y., 1337

Johansen, E., 322
Johansson, T., 202

Jones, J. G., 152

Jordana, X., 34

Joshi, S., 383

Judelson, H. S., 330

Jurkowski, G. I., 511

Kahn, D., 292

Kaido, M., 967

Kaloshian, I., 55

Kaneda, T., 986

Kang, L., 1328

Kannenberg, E. L., 283

Kaplan, I., 571

Katagi, C., 583

Katinakis, P., 1283

Kawaguchi, M., 1223

Keen, N. T., 999

Kemp, G., 888

Keshavarzi, M., 805

Khatib, M., 272

Kijne, J. W., 816

Kim, J. F., 644

Kim, W. S., 1259

Kimura, E. T., 827

Kinter, M., 1019

Király, L., 976

Kiss, E., 235

Kitajima, J. P., 827

Klebsiella pneumoniae, on wheat, nitrogen fixation, 1078

Klessig, D. F., 511

Kloek, A. P., 162

Kluepfel, D. A., 1376

Knogge, W., 1103, 1114

Kobayashi, H., 1153

Kobayashi, K., 475

Kobayashi, M., 749

Kogel, K.-H., 118, 304, 366, 374, 484

Kohel, R. J., 1234

Koiwa, H., 780

Komeda, K., 1355

Kopka, C., 789

Kouchi, H., 1223

Kovács. I., 343

Krajinski, F., 1063

Krause, M., 131

Krishnan, H. B., 1259

Krol, E., 292

Kubo, Y., 749

Kuiper, I., 6, 1185

Kunkel, B. N., 162, 313

Kuramae, E. E., 827

Küster, H., 62, 292, 1063

Lagendijk, E. L., 6, 1185

Lahey, K. A., 1394

Lamers, G. E. M., 1185

Lane, L. C., 976

Langen, G., 374

Latour, X., 1269

Lauber, E., 292

Lavie, M., 931

Leach, J., 1146

Leach, L. E., 771

Lee, S.-W., 602

Lees, A. K., 880

Lei, Z., 1306

Leifsonia xyli

— circular genome, cover photo, August

-on sugarcane: functional genomics platform, 175; genome sequence, 827

Lemanceau, P., 1201

Lemos, E. G. M., 827

Lemos, M. V. F., 827

Le Quéré, A., 202

Letelier, I., 34

Leung, D., 394 
Leung, H., 1146

Li, L., 547

Liang, D., 571

Liebe, C., 292

Lim, M. T. S., 313

Linde, C. C., 1114

Lindow, S. E., 521, 999

Littlefield, L. J., 739

Liu, B., 1146

Liu, J., 583

López-Baena, F. J., 676

Lopez de Leon, A., 593

Lorang, J. M., 577

Lorio, J. C., 1259

Losada, L., 254

Lotus japonicus

—nodule development, potassium transporter, induction, 789

-nodules: gene analysis, 1223; polyamine biosynthesis, 1283

Lübeck, J., 1126

Luck, J., 224

Lugtenberg, B. J. J., 6, 557, 1185

Lundeberg, J., 202

Lycopersicon hirsutum (see also Tomato)

- Potato virus $Y$, recessive resistance, genomelinked protein, 322

Lyngkjær, M. F., 109, 729

MacFarlane, S., 1086

Machado, M. A., 827

Madinabeitia, N., 676

Mäe, A., 1366

Magnaporthe grisea

-PAK kinase genes, functions, 547

-on rice: defense gene predictors, resistance, 1146; sequence tag analysis, 1337

Maize, rust resistance gene, processing on wheat and barley, aberrant mRNA, 853

Makart, S., 90

Manners, J., 224

Manning, V. A., 491

Mansfield, J., 805, 1039, 1250

Manthey, K., 1063

Manuscript, checklist, 128

Marie, C., 958

Marino, C. L., 827

Marmeisse, R., 1029

Martin, G. B., 1212

Martinez, J. P., 467

Martinez de Ilarduya, O., 55

Mattinen, L., 1366

Mauleon, R., 1146

Mauro, S. M. Z., 827

Mayama, S., 81, 245

McCouch, S. R., 1348

McDonald, B. A., 1114

McFadden, H., 654

Medicago spp.

- M. sativa, nodules, superoxide dismutases and hydrogen peroxide, 1294

-M. truncatula: Colletotrichum trifolii, compatible and incompatible interaction, analysis, 909; mycorrhizal establishment, dmi3 gene, 1385; root endosymbioses, profiling in nodules and arbuscular mycorrhiza, 1063; Sinorhizobium meliloti symbiosis, stress adaptation gene typA, 235

Medina, C., 676

Meek, D. J. J., 1318

Megessier, S., 414

Mei, M., 1146

Meilhoc, E., 292

Melayah, D., 1029

Meloidogyne spp., on tomato

—defense response, salicylic acid, 351

- Mi-mediated resistance, 532

Melon necrotic spot virus, resistance in melon and nonhosts, molecular characterization, 668

Menck, C. F, 827

Mew, T., 1146

Mihlan, M., 383

Minsavage, G. V., 152

Mise, K., 967

Miyaki, C., 827

Molina, A., 763

Monteiro-Vitorello, C. B., 827

Morel, C., 322

Mori, T., 245

Moriones, E., 668

Morozov, S. Y., 921

Moury, B., 322

Moy, P., 1051

Mudgett, M. B., 633

Mudgett, M. G., 152

Muñiz, M., 55

Murphy, R. M., 175

Muthukrishnan, S., 1306

Mycorrhizae

- arbuscular, Medicago truncatula, transcriptome profiling in nodules, 1063

- Medicago truncatula, elicitation and dmi3 gene, 1385

- Paxillus involutus on Betula pendula, transcriptional response, 202

- tomato mutant, defense-related genes, 1103

-Vicia faba, leghemoglobin gene, root nodules and arbuscule, 62

Mycotoxin

-Ptr ToxA, Pyrenophora tritici-repentis, multiple motifs, 491

- victorin: Arabidopsis thaliana, characterization, 577; biosynthesis in oat, 81 Mysore, K. S., 1328, 1306

Nagel, R. J., 175

Nakashima, M., 749

Nakayashiki, H., 81, 245

Nandi, A., 623

Narusaka, M., 749

Narusaka, Y., 749

Nathues, E., 383

Navarre, D. A., 351

Navarre, R., 140

Nelson, R. S., 583

Newton, J. A., 880

Nicolaisen, M., 1175

Nicole, M., 805, 1039

Nicotiana spp. (see also Tobacco): Cucumber mosaic virus, necrotic pathotype, amino acid determinant, 837; Tomato leaf curl virus, gene expression, 27

$-N$. benthamiana, programmed cell death, Pseudomonas type III effector, 1328

-N. edwardsonii, virus resistance, PR-1 and single gene, 976

-N. tabacum, Tobacco mosaic virus, protein association, replication and RNA silencing, 583

Nieto, C., 668

Nishimura, M., 547

Nitrogen-activated protein kinases, Sclerotinia sclerotiorum, sclerotial development regulated by, 404

Nodulation

-alfalfa, abnormalities, transgenes, 16

-Lotus japonicus: gene analysis, 1223; polyamine biosynthesis, spatial organization, 1283; potassium transporter of plasma membrane, 789

-Rhizobium spp.: assay, activating compounds, 816; flavonoids and indole-3-acetic acid synthesis, 1153; key regulator, type IIIdependent protein secretion, polysaccharide synthesis, 958
- superoxide dismutases and hydrogen peroxide, localization, 1294

Nombela, G., 55

Nostoc, cyanobacterial genes, heterocysts and dinitrogen fixation, symbiosis profile, 436

Nunes, L. R., 827

Oat

- defense response, hypersensitivity and apoptosis, nitric oxide and oxygen, 245

-phytoalexin biosynthesis, transferases involvement, analysis, 81

—Victoria blight, victorin sensitivity, 577

O'Connell, R., 272

Oesch, N. W., 467

Oeser, B., 383

Oh, H.-S., 447

Ojanen-Reuhs, T., 958

Okinaka, Y., 999

Okuno, T., 967, 1355

Oliveira, R. C., 827

Oliver, R. P., 456

Ollero, F. J., 676

Omarov, R. T., 195

Ongena, M., 1009

Orange, N., 1269

Ortori, C., 880

Osmotin, cytotoxicity to Saccharomyces cerevisiae, abolished by K, 780

Ott, T., 789

Paal, J., 1126

Pacheco, M. G., 853

Pak, K., 254

Palloix, A., 322

Palukaitis, P., 571

Pan, H., 1337

Panicum mosaic virus, capside protein, symptoms elicited on nonhost plant, gene silencing, 263

Panopoulos, N. J., 1250

Papanice, M. A., 98

Parada, M., 676

Pardo, J. M., 780

Park, P., 245, 749

Park, R. F., 853

Pathotoxin, alicidin, Xanthomonas albilineans, PKS and NRPS genes, 414

Patriarca, E. J., 720, 1043

Pavet, V., 343

Paxillus involutus, on Betula pendula, ectomycorrhizae, transcriptional response, 202

Pea

—nodulating strain, lipopolysaccharides, bacteroid formation, 283

—nodule symbiosomes, Rhizobium bacteroids, cell surface interactions, 216

—pisatin, fungal response, 798

Peñaloza-Vázquez, A., 162

Pépin, R., 1029

Pepper (see also Capsicum annuum)

-Xanthomonas campestris, lipopolysaccharides for defense, suppression, 805, 1039

Pereira, G. G., 827

Perlick, A. M., 62, 1063

Perl-Treves, R., 70

Perna, N. T., 999

Perret, X., 958

Petrasovits, L A., 175

Pfund, C., 696

Phaseolus vulgaris (see also Bean)

-Aspergillus niger, polygalacturonase activators, 888

- ectopic roots, abortive nodule primordia, 1043

-P. syringae, Psy61 effector, 254

Phytoalexin 
- biosynthesis in oat, transferases involvement, 81

-Erwinia amylovora on Rosaceae, virulence, efflux pump, 43

- pisatin, genes reduced production, responses, 798

Phytophthora spp.

-P. infestans: on potato, quantitative trait loci, gene markers, 1126; potato, resistance, $R$ gene linkage, 428; zoosporogenesis, gene induction, pathways, 330

- $P$. sojae, on soybean: avirulence requires elicitor and regulator, resistance gene, 394; gene expression patterns, 1051

Phytoplasma: distribution in infected plants, bioimaging and PCR, 1175; cover photo, November

Phytoremediation, plant-microbe interaction, review, 6

Phytotoxin, coronatine, Pseudomonas syringae

- genes, temperature, 1095

- on tomato, biosynthetic mutants, 162

Pieretti, I., 414

Pieterse, C. M. J., 895

Pinus pinaster, Hebeloma cylindrosporum, mutants obtained through insertional mutagenesis, 1029

Pirhonen, M., 1366

Pisum sativum, nodules, superoxide dismutases and hydrogen peroxide, 1294

Poinsot, V., 235

Polymyxa graminis, vector for Soilborne wheat mosaic virus and Wheat spindle streak mosaic virus, RNA and coat protein in resting spores, 739

Potato

-blackleg, infection and lactones, 1269

-Erwinia carotovora: pooled transposon mutation grid, effector and helper proteins, 943; susceptibility increased by lactones, 880

-late blight, quantitative trait loci, gene markers, 1126

Potato leafroll virus, yeast homologous recombination system, mutagenesis and chimeric viruses, plant virus analysis, 571

Potato mop-top virus, TGBp1 trafficking, requirements, encoded genes, 921

Potato virus A, on Nicotiana spp., 6K 2 protein, symptom-inducing induction function, 502

Potato virus $Y$, in pepper and tomato, virulence and genome-linked protein, mutations, 322

Potyviruses, $6 \mathrm{~K} 2$ protein, long-distance movement and symptoms, host specificity, 502

Prinsen, E., 1153

Prior, P., 931

Programmed cell death (see Apoptosis)

Prospéri, J.-M., 909

Pryor, A. J., 853

Pseudomonas spp.

- P. brassicacearum, phenotypic variation, root-colonization strategy, 872

-P. chlororaphis, phenazine-1-carboxamide production, factors for biocontrol, 557

-P. fluorescens: antibiotic from, Fusarium oxysporum affected by, 1201; Fusarium oxysporum hyphae colonized by, chemotaxis, 1185

- P. oxysporum, on tomato, bacterial colonization, chemotaxis, 1185

- P. putida, on bean, lipoxygenase pathway, systemic resistance, 1009

-P. syringae: on Arabidopsis, hypersensitivity, 357; on Arabidopsis, incompatible interaction, proline accumulation and AtP5CS2 gene activation, 343; "defense, no death" phenotype, hypersensitivity, 511; effector protein, tobacco genes and virulence,
1139; epiphytic fitness, AHL synthesis, regulation, 521; HopPtoF locus, chaperone and cognate effector encoded, 447; on Nicotiana benthamiana and tomato, programmed cell death, 1328; phytotoxin coronatine, synthesis genes, temperature, 1095; Psy61 effector, virulence and avirulence, HrpL-dependent, 254; syringolin A synthesis, gene analysis, 90; on tomato, coronatine biosynthetic mutants, characterization, 162; type III secretion system, transcriptional regulation, 1250; virulence and avirulence activities, mutations, RIN4 disappearance, 313

Puccinia spp.

- P. sorghi, on maize, aberrant processing in wheat and barley, 853

-P. striiformis, on wheat, growth-stagespecific resistance, 1242

Pühler, A., 62, 292, 1063

Pujol, C. J., 521

Pyrenophora tritici-repentis

-host-selective toxin gene, schematic analysis, cover photo, May

- pathogenic and nonpathogenic isolates, hostselective toxin gene, 467

- on wheat, necrosis-inducing toxin Ptr ToxA, multiple motifs, 491

Qiu, W., 263

Quandt, H.-J., 62

Quiñones, B., 521

Qutob, D., 1051

Raaijmakers, J. M., 120

Raffier, C., 1029

Ralstonia solanacearum

—on Arabidopsis thaliana, flagellin as elicitor, 696

- phylogeny, type III-dependent effectors, correlation, 931

-swimming motility, regulation by FlhDC and environment, 686

-on tobacco, avirulence gene diversity and wilt, 1376

Randles, J. W., 27

Rangaswamy, V., 162

Raymundo, A., 771

Redondo-Nieto, M., 216

Resistance

-Arabidopsis to Colletotrichum higginsianum, RCJ1 locus, 749

-Arabidopsis to Fusarium oxysporum, ethylene response factor, 763

-Arabidopsis, systemic, rhizobacteria-induced, 895

-Arabidopsis thaliana to Albugo candida, gene dependence, 711

-barley to powdery mildew: enhanced by mutant, 374; inappropriate host, 484

-bean to nonpathogenic Pseudomonas, systemic, 1009

-Cauliflower mosaic virus, transactivator/viroplasmin, virulence, 475

- gene transfer: between species, maize, wheat, and barley rusts, 853 ; intergeneric, allelespecific activation, 224

-maize to: rice pathogen, nonhost reaction, 771 ; rust, aberrant mRNA processing in wheat and barley, 853

- melon to Melon necrotic spot virus, molecular characterization, 668

- Mi-1-mediated, rme1, 55

—phytoalexin production, mechanism, 798

- potato to: Erwinia carotovora, lactones, 880 ;

Phytophthora infestans, linked $R$ genes, 428

-quantitative, wheat and barley, germinlike protein GLP4, 109 -recessive, Capsicum annuum and Lycopersicon hirsutum, genome-linked protein, 322

-rice to: bacterial blight, novel gene $x a 5,1348$; blast, defense genes as predictors, 1146

- tobacco to Tobacco mosaic virus, ethylene response transcription factor, 1162

- tomato to: aphids and nematodes, $\mathrm{Mi}$ mediated, 532; bacterial canker, proteomic analysis, 1019

Reuhs, B., 958

Review, rhizoremediation, beneficial plantmicrobe interaction, 6

Reviewers, acknowledgment of, 3

Rezende, J. A. M., 195

Rhizobacteria, transcriptome, Arabidopsis resistance induced by, 895

Rhizobium spp.: indole-3-acetic acid synthesis flavonoids, 1153; key regulator, type IIIdependent protein secretion, polysaccharide synthesis, 958; nodulation assay, lipochitin oligosaccharides and NodD-activating compounds, 816

-R. etli: glutamine utilization, 720; nodule primordia, abortive ectopic roots, cover photo, October; 1043

- R. leguminosarum: pea-nodulating strain, lipopolysaccharides, bacteroid formation, 283 ; in pea nodules, cell surface interactions, 216

Rhizoctonia solani, on Arabidopsis, GSTF8 promoter for strains, 70

Rhizoremediation, plant-microbe interaction, review, 6

Rhynchosporium secalis, on barley, virulence mechanisms, genetic analysis, 1114

Riccio, A., 720

Rice

-Acidovorax avenae, immune response elicited, 986

-bacterial blight: two-component regulatory system, AvrXa21 activity, 602; type III effectors, virulence determinants, 1192

-blast: PAK kinase genes, functions, 547; resistance, defense genes, predictors, 1146; sequence tag analysis, 1337

-immunity, mediated innate response, bacterial genes, 593

- overexpression of (At)NPRl, cell death phenotype, 140

-resistance gene xa5, novel resistance, 1348

Rickert, A. M., 1126

Rimmer, S. R., 711

Rivilla, R., 216

Robertson, A.E., 1376

Robinson, A. F., 1234

Roden, J., 633

Rodríguez-Navarro, D.-N., 676

Rodríguez-Palenzuela, P., 184

Rogato, A., 1043

Rojas, C. M., 184, 644

Ronald, P. C., 140, 593, 602

Rooke, L., 853

Ross, K., 976

Rossignol, F., 1009

Rossiter, J., 805, 1039

Rott, P. C., 414

Royer, M., 414

Rozenbaum, I., 254

Rozwadowski, K., 711

Rüberg, S., 292

Rubio, M. C., 1294

Ruiz-Sainz, J. E., 676

Saccharomyces cerevisiae, mannosylation mutants, cytotoxicity of osmotin, 780

Sakamoto, M., 245

Sakurai, T., 749 
Salánki, K., 837

Saleh, S. S., 865

Salicylic acid

-Arabidopsis, responsive genes, 34

- Cucumber mosaic virus, resistance enhanced, independent mechanism, 623

-Pseudomonas syringae, "defense, no death" mutants, 511

- tomato root-knot nematodes, defense response, 351

Salzman, R. A, 780

Sanchez, L., 1385

Sanchez, P., 456

Sandgren, M., 921

Satou, M., 749

Savenkov, E. I., 921

Savin, A., 414

Schäfer, P., 366

Schechter, L. M., 644

Schenk, A., 1095

Schneider-Pokorny, J., 90

Schoelz, J. E., 976

Scholthof, H. B., 195

Scholthof, K.-B. G., 263

Schorn, C., 537

Schouten, A., 1201

Schultheiss, H., 484

Schulz, A., 1175

Schulze Gronover, C., 537

Schürch, S., 1114

Schweizer, P., 109

Sclerotinia sclerotiorum

- sclerotial development, regulation linked with $\mathrm{pH}$ and cAMP sensing, 404

-on soybean, polygalacturonase, oxalate, and $\mathrm{pH}$ effect, 1402

Secale cereale, ergot, oxidative stress response, transcription factor, 383

Seilaniantz, A. R., 1139

Seki, M., 749

Sekine, K.-T., 623

Sella, L., 1402

Selth, L. A., 27

Sessa, G., 1212

Setúbal, J. C., 827

Seunes, B., 931

Shah, G., 532

Shah, J., 623

Shakya, R., 90

Shan, L., 447

Shan, W., 394

Shapiro, A. D., 357

Sharma, S. B., 511

Shen, Y., 593, 602

Sherrier, D. J., 283

Shinogi, T., 245

Shinozaki, K., 749

Shiraishi, T., 749

Simpson, A. J. G., 827

Singh, K. B., 70

Sinorhizobium spp.

- S. fredii: on soybean, cultivar-specificity protein, type III protein, 1259; symbiotic signals, NoIR regulation, 676

-S. meliloti: gene expression in microtoxic and symbioic conditions, 292; malate dehydrogenase gene, polar effects, 1318; on Medicago truncatula, symbiosis, stress adaptation gene typA, 235; on sweetclover, expansin gene upregulated, 613

Siqueira, W., 827

Skøt, L., 729

Smadja, B., 1269

Smith, F. A., 1103

Smith, P. H., 1242

Smith, R. K., Jr., 511

Smith, S. E., 1103

Söderström, B., 202
Soilborne wheat mosaic virus, movement protein and RNA in spores of vector, 739

Solomon, P. S., 456

Solovyev, A. G., 921

Soria-Díaz, M. E., 676

Souche, S., 322

Soybean

-cultivar-specificity protein, from

Sinorhizobium fredii, 1259

- Phytophthora sojae, gene expression patterns, 1051

-Sclerotinia sclerotiorum, polygalacturonase, oxalate, $\mathrm{pH}$ interaction, 1402

Soylu, S., 805, 1039

Spanu, P. D., 1250

Spetz, C., 502

Spring beauty latent virus, on Arabidopsis thaliana, SSB1 locus, 967

Sreedharan, A., 162

Sreenivasaprasad, S., 272

Stagonospora nodorum, on wheat, pathogenicity and $\mathrm{G} \alpha$ subunit, 456

Stall, R. E., 152

Stanton, L., 888

Steinau, M., 853

Steinberg, C., 1201

Steven, B., 1318

Stevens, C., 1250

Stockinger, E. J., 1019

Strahwald, J., 1126

Stratford, R., 1242

Stubner, S., 1095

Suganuma, N., 1223

Sugarcane

-leaf scald, pathotoxin alicidin production, 414

-Leifsonia xyli: functional genomics platform, 175; genome sequence, 827

Sumner, L. W., 1306

Suppression subtractive hybridization, Botrytis cinerea, gene up-regulation during infection, 537

Sussan, T., 254

Sweetclover, Sinorhizobium meliloti, expansin gene upregulated, from Melilotus alba, 613

Syringolin, Pseudomonas syringae, synthesis and genes, 90

Szabados, L., 343

Tada, Y., 245

Tak, T., 816

Takahashi, H., 623

Takahashi, S., 245

Takano, Y., 1355

Takayama, S., 986

Takayanagi, N., 1355

Takita, M. A., 827

Tan, K.-C., 456

Tanaka, N., 986

Tang, X., 447, 1139, 1328

Tani, S., 330

Tans-Kersten, J., 686, 696

Tatè, R., 720, 1043

Tenberge, K. B., 383

Thara, V. K., 1139

Theunis, M., 1153

Thomas, T., 1337

Thon, M., 1337

Thonart, P., 1009

Thordal-Christensen, H., 109

Thwaites, R., 1250

Timmer, L. W., 1394

Tobacco (see also Nicotiana spp.)

-bacterial wilt, avirulence gene diversity, 1376

- effector protein as virulence factor, genes, 1139

-transgenic, flax rust resistance gene $L, 224$

Tobacco mosaic virus

-126-kDa protein, virus replication association, RNA silencing, 583
- ethylene response transcription factor, resistance enhanced, 1162

- gene silencing delay, encoded suppressor, cover photo, June

-on Nicotiana edwardsonii, enhanced resistance, 976

Tobacco necrosis virus, on Nicotiana edwardsonii, enhanced resistance, 976

Tomato (see also Lycopersicon hirsutum)

- arbuscular mycorrhizal development, defense-related genes, 1103

-bacterial canker, proteomic analysis, 1019

-bacterial leaf spot, host-parasite interaction and importance of $o p g H_{\mathrm{xcv}}, 152$

- Cucumber mosaic virus, transgenic resistance, mechanisms, 98

- foot and root rot, chemotaxis of Pseudomonas fluorescens to Fusarium oxysporum, 1185

- gene $M i-1$, resistance, nematodes, aphids, and whitefly, 55

- gene profiling, resistance response to Xanthomonas campestris, 1212

- programmed cell death, Pseudomonas type III effector, 1328

-resistance genes to nematodes and aphids, Mi-mediated, 532

-root-knot nematode, defense response and root-knot nematode, 351

Tomato bushy stunt virus

—defective interfering RNAs, host-specific, 195

-on Nicotiana edwardsonii, enhanced resistance, 976

Tomato leaf curl virus, host response, gene expression, 27

Torregrosa, C., 909

Tosa, Y., 81, 245

Toth, I. K., 880, 943

Trick, H. N., 771, 853

Trinh, H. X., 81

Triplett, E. W., 1078

Trippe, A. F., 491

Truffi, D., 827

Trujillo, M., 304

Truniger, V., 668

Tsai, S. M., 827

Tshuikina, M., 1366

Tudzynski, B., 537

Tudzynski, P., 383

Tunlid, A., 202

Tyler, B. M., 394

Tytga, T., 846

Udvardi, M. K., 789, 1283

Ueng, P. P., 1394

Uhlén, M., 202

Ullman, D. E., 532

Ullrich, M. S., 43, 1095

Uquillas, C., 34

Valkonen, J. P. T., 502, 921

van Brussel, A. A. N., 816

Vance, C. P., 1294

Van Criekinge, W., 846

van den Berg, G., 1201

van Eck, H. J., 428

VanEtten, H. D., 798

Vanholme, B., 846

Vanhoutte, I., 846

van Loon, L. C., 895

van Rij, E. T., 557

Van Sluys, M. A., 827

van Spronsen, P. C., 816

Vectors, yeast-bacteria shuttle, plant-virus analysis, Potato leafroll virus, 571

Vedam, V., 283

Verchot-Lubicz, J., 739

Verhagen, B. W. M., 895

Viard, M., 414 
Vicia faba, leghemoglobin gene, endomycorrhiza symbiosis, 62

Vieweg, M. F., 62

Vinardell, J. M., 676

Visser, R. G. F., 428

Vivien, E., 414

Vleeshouwers, V. G. A. A., 428

von den Driesch, M., 383

Walker, N. R., 739

Wang, C.-M., 436

Way, H., 224

Wechter, W. P., 1376

Weidmann, S., 1385

Weidner, S., 292

Weingart, H., 43, 1095

Werij, J. S., 428

Wesselink, M., 557

Wheat

— growth-stage-specific resistance, biotrophic pathogens, 1242

-nitrogen fixation, Klebsiella pneumoniae, 1078

-quantitative resistance, germinlike protein GLP4, 109

-Stagonospora nodorum on wheat, pathogenicity and $\mathrm{G} \alpha$ subunit, 456

- $\tan$ spot, pathogenicity and toxin gene, 467

- transgenic line, apoplastic extracts, 1306

Wheat spindle streak mosaic virus, coat protein in spores of vector, 739

Whisson, S. C., 943
White, F. F., 1192

Wiggins, B. E., 976

Willard, B., 1019

Williams, A., 1086

Williams, P., 880

Williamson, V. M., 55, 351, 532

Wilson, I. W., 654

Wolpert, T. J., 577

Wood, D., 827

Worland, A. J., 1242

Wu, Q., 798

Wu, S., 1146

Xanthomonas spp.

-X. albilineans, alicidin production pathotoxin, PKS and NRPS genes, 414

-X. campestris: in pepper, lipopolysaccharides, defenses, 805, 1039; on pepper and tomato, AvrXv4 effector, cell translocation, 633; on tomato, gene profiling, resistance response, 1212; on tomato, host-parasite interaction, importance of $\mathrm{opgH}_{\mathrm{xcv}}, 152$; on tomato leaflets, symptoms, cover photo, February

$-X$. oryzae, on rice: genes in secretion and sulfation, immune response, 593; nonhost reaction on maize, resistance gene, 771; twocomponent regulatory system, AvrXa21 activity, 602; virulence determinants, type III effectors, 1192; Xa5 resistance gene, cover photo, December

$\mathrm{Xu}$, J.-R., 547

$\mathrm{Xu}, \mathrm{Z} ., 1234$
Xue, C., 547

Xue, Q., 602

Yadav, R. C., 593

Yamamoto, A., 1223

Yamauchi, J., 1355

Yang, A., 1086

Yang, B., 1192

Yang, C.-H., 999

Yang, Q., 81, 1146

Yang, S., 999

Yang, Y., 1139

Yao, N., 245

Yarden, O., 394

Yatzkan, E., 330

Yu, I., 511

Yu, J. Z., 1234

Yuan, R., 1394

Zamyatnin, A. A., Jr., 921

Zanca, A. S., 827

Zeyad, S., 254

Zhang, C., 357

Zhang, H.-B., 1234

Zhang, K., 81

Zhang, S., 1146

Zhao, B., 771

Zhou, J.-M., 447, 1139

Zhu, T., 895

Zhu, X., 1146

Zimmermann, G., 109 